\title{
Estratégia\&Negócios
}

ISSN 1984-3372

http://www.portaldeperiodicos.unisul.br/index.php/EeN/

\section{ANÁLISE DA TRANSMISSÃO DE PREÇOS NO SETOR SUCROALCOOLEIRO BRASILEIRO}

\section{PRICE TRANSMISSION ANALYSIS IN BRAZILIAN SUGARCANE SECTOR}

\section{Alexander Block}

Universidade Federal de Santa Maria - UFSM.

E-mail: lexblock@hotmail.com

\section{Daniel Arruda Coronel}

Professor Adjunto do Mestrado em Administração da Universidade Federal de Santa Maria - UFSM. Doutor em Economia Aplicada pela Universidade Federal de Viçosa - UFV.

E-mail: daniel.coronel@ufv.br

\section{Gilberto de Oliveira Veloso}

Professor Associado do Programa de Pós-Graduação em Economia da Universidade Federal de Santa Maria - UFSM. E-mail: gilbertovel@gmail.com

Recebido em 21/05/2012. Aprovado em 11/07/2012. Disponibilizado em 01/10/2012.

Avaliado pelo Sistema double blind review

R. eletr. estrat. neg., Florianópolis, v.5, n.2, p. 120-137, mai./ago. 2012

http://portaldeperiodicos.unisul.br/index.php/EeN/index
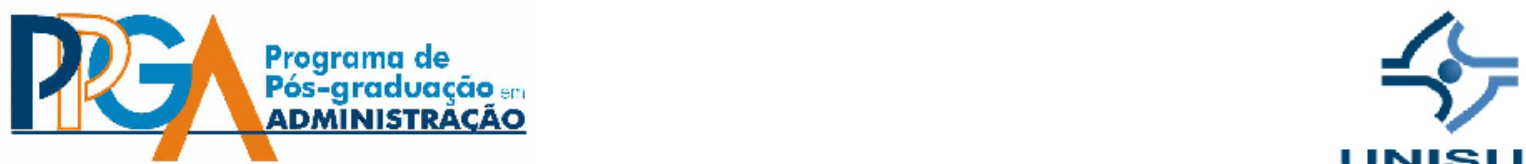

UNISUL

CCopyright 2008 UNISUL-PPGA/Estratégia e Negócios. Todos os direitos reservados. Permitida citação parcial, desde que identificada a fonte. Proibida a reprodução total. Em caso de dúvidas, consulte o editor:

ademar.unisul@gmail.com; (48) 3229-1932. 


\section{RESUMO}

O objetivo deste trabalho é analisar o processo de transmissão de preço no setor sucroalcooleiro, buscando relações entre as séries de preços estudadas para explicar suas oscilações e as influências de cada produto na formação do seu próprio preço e do preço dos demais e, dessa forma, verificar a adaptabilidade da Lei do Preço Único dentro do setor. Para isso, foi realizada uma pesquisa de caráter quantitativo descritivo por meio de dados secundários, utilizando séries de preços de cana-deaçúcar, etanol hidratado e açúcar no Estado de São Paulo, no período de fevereiro de 1999 até setembro de 2010. Esses dados foram analisados segundo o modelo de Autorregressão Vetorial (VAR), e os resultados obtidos demonstram que o preço destes produtos tem forte ligação, destacando-se o caso do etanol hidratado, que influencia fortemente o preço dos demais, mas não sofre qualquer influência das oscilações de preço da cana-de-açúcar e do açúcar.

Palavras-chave: Transmissão de preços. Cana-de-açúcar. Etanol. Açúcar.

\section{INTRODUÇÃO}

O setor sucroalcooleiro do Brasil movimenta significativamente a economia brasileira, pois, de acordo com dados da safra 2010/2011, a produção do setor está em 55 bilhões de reais, gerando 4,5 milhões de empregos diretos e indiretos. Além disso, 0 Brasil exporta 20 milhões de toneladas de açúcar, de 32 milhões de toneladas que são produzidas no país. A capacidade de moagem, por sua vez, corresponde a 560 milhões de toneladas de cana utilizadas para produzir 32 milhões de toneladas de açúcar e 27 bilhões de litros de álcool. A exportação de açúcar foi de 9 bilhões de dólares, e a exportação de álcool, de 2,2 bilhões de dólares. Destaca-se também que o setor sucroalcooleiro recolhe 13 bilhões em impostos. O Valor Bruto da Produção, que é a soma do valor das principais lavouras do país, está estimado em $\mathrm{R} \$ 218,63$ bilhões em 
2012, segundo a Assessoria de Gestão Estratégica do Ministério da Agricultura, Pecuária e Abastecimento (JORNALCANA, 2012).

Conforme Costa, Burnquist e Guilhoto (2006), desde a segunda metade da década de 1990, o setor sucroalcooleiro do Brasil tem se mantido na posição de maior produtor e exportador mundial de açúcar e maior produtor mundial de álcool combustível de cana. Dentre os fatores que contribuem para a posição de destaque do Brasil no mercado internacional do açúcar e álcool, estão os baixos custos de produção.

A cana-de-açúcar brasileira tem como característica de destaque a possibilidade de cultivo em praticamente todo o ano e com custos altamente competitivos. Outra característica peculiar em relação à análise de aspectos de produção do setor sucroalcooleiro brasileiro é a sua concentração em duas grandes regiões: a região Centro-Sul e a região Norte-Nordeste. De acordo com dados do Ministério da Agricultura, Pecuária e Abastecimento, em julho de 2008, o segmento da agroindústria canavieira no Brasil contava com 396 unidades produtoras de açúcar e álcool em atividade, sendo 315 na região Centro-Sul e 81 unidades no Norte-Nordeste (BRASIL, 2008).

A região Centro-Sul assume uma importância destacada tanto em termos de produção como de área plantada de cana-de-açúcar. A participação relativa da Região Centro-Sul ultrapassou $80 \%$ do total da produção nacional de cana nas últimas safras, e o Estado de São Paulo foi o maior produtor no âmbito da região Centro-Sul e no âmbito nacional (responsável por mais de $60 \%$ da produção brasileira e mais de 70\% da produção da região Centro-Sul, na safra 2000/01).

Em 2011, a Agência Nacional do Petróleo (ANP) informou que o Brasil produziu cerca de 28,2 milhões de metros cúbicos de etanol hidratado e anidro no ano de 2010, sendo o segundo maior produtor mundial de etanol, atrás dos Estados Unidos, e o maior exportador. A região Sudeste se destaca como a maior produtora de etanol no Brasil, sendo responsável por $66,9 \%$ da produção nacional.

Tendo em vista a relevância do setor, o presente estudo busca verificar se há aplicabilidade da Lei do Preço Único dentro de um setor e, dessa forma, observar se 
os principais produtos deste setor apresentam um equilíbrio de longo prazo, ou seja, como se dá o processo de transmissão de preços entre os produtos.

Considerando a importância do setor açúcar-alcooleiro, este trabalho tem o objetivo de estudar as relações existentes entre o preço da cana-de-açúcar, o etanol hidratado e o açúcar, buscando relações entre as séries de preços estudadas que expliquem as oscilações de preço e as influências de cada um desses produtos na formação do seu próprio preço e do preço dos demais. Assim, os participantes que atuam no processo de negociação dentro do setor terão informações que subsidiem o processo decisório por meio de uma ferramenta de análise que possa ser empregada em negociações futuras.

O artigo está estruturado em três seções, além desta introdução. Na segunda seção, são apresentados os procedimentos metodológicos; na terceira seção, os resultados obtidos são analisados e discutidos e, por fim, são apresentadas algumas considerações finais.

\section{REFERENCIAL TEÓRICO}

A transmissão de preços está associada à teoria da Lei do Preço Único (LPU). De acordo com Balcombe e Morrison (2002), o processo de transmissão de preços fundamenta-se nas hipóteses de que o movimento de preços e o ajustamento integral resultam em transmissão completa em algum ponto de tempo; na dinâmica e velocidade de ajustamento; e na assimetria de respostas, isto é, se as variações de preços são transmitidas assimetricamente entre mercados.

Segundo Krugman e Obstfeld (2005), os bens idênticos comercializados em diferentes regiões devem ser vendidos pelo mesmo preço, quando seus preços são expressos em termos da mesma moeda, em mercados sob livre concorrência e na ausência de custos de transporte e barreiras oficiais ao comércio. 
A LPU tem sido amplamente defendida na literatura, porém, estudos como os de Fackler e Goodwin (2001) sinalizam que os pressupostos teóricos da LPU são muito restritivos, já que não incluem outras variáveis como custos de transação e volume comercializado entre mercados, que também desempenham papel importante na análise de integração de mercados.

Para a realização deste estudo, considerou-se verificar a aplicabilidade da LPU dentro de um único setor, mas, testando a sua validade em produtos diferentes. Essa aplicabilidade a um setor pode trazer implicações que determinem a dinâmica de relacionamento de preços entre os produtos estudados e, assim, determinar o nível de integração entre eles. A adaptação da LPU dentro do setor sucroalcooleiro, no contexto de transmissão de preço, pode determinar como variações no preço do biocombustível etanol são repassadas a alimentos como o açúcar e a produtos agrícolas como a cana-de-açúcar.

A produção de biocombustíveis tem gerado uma série de questões a respeito de segurança alimentar. O mercado de commodities agrícolas é também um importante determinante na dinâmica do mercado de biocombustíveis. Com essa motivação, Tokgoz e Elobeid (2006) pesquisaram as relações entre os mercados de etanol, cana-de-açúcar e milho. Os estudos revelam competição entre o setor de etanol e os outros setores que utilizam a mesma matéria-prima, de modo que a lucratividade relativa desses setores irá determinar as tendências de longo prazo no setor agrícola.

Em seu estudo a respeito dos impactos do uso de biocombustíveis, (VIDOSH; PRAKSH; ALOK, 2011) relata os benefícios do desenvolvimento deste setor. Segundo ele, os biocombustíveis, além de promover redução nas emissões de poluentes, podem ser um fator de desenvolvimento regional ao trazerem novas fontes de renda. Apesar disso, possuem o poder de influenciar aumentos nos preços de alimentos ao ocuparem áreas destinadas à produção destes.

Os estudos de Silva e Almeida (2006) apontam a existência de uma forte ligação entre os preços do petróleo e do açúcar, passando pela produção de etanol de 
açúcar. Nesse sentido, o Brasil tem um papel importante pela sua importância nos mercados internacionais de açúcar e etanol.

Dong (2007) defende a ideia de que os países em desenvolvimento devem considerar o problema de segurança alimentar em face da expansão dos biocombustíveis. Segundo o autor, quanto mais terra for alocada para a produção de matéria-prima para biocombustíveis, maiores serão os preços dos grãos.

A transmissão de preços envolvendo combustíveis no Brasil ainda é um tema pouco abordado. Porém, podem-se encontrar algumas análises referentes à assimetria. Gamarra (2009) analisa o processo de transmissão de preços entre os mercados do etanol e da gasolina desde o lançamento dos carros "flex". Por intermédio de modelos com vetores de correção do erro (VEC) e de testes de causalidade, observa-se haver relações de curto e longo prazo e afirma-se a integração entre os mercados. A assimetria na transmissão de preços do óleo diesel é estudada por Canêdo-Pinheiro (2011), que, por meio do modelo VEC com quebra estrutural, constata que assimetria se manifesta no curto e no longo prazo.

Para a realização do presente estudo, foi utilizado um modelo de transmissão de preços a fim de verificar a origem das variações de preço dos produtos relacionados nessa cadeia. As análises de transmissão de preços pretendem responder às seguintes questões: a) em que nível de mercado se origina as variações nos preços e em que sentido essas variações se transmitem; b) durante que período se dá a transmissão e com qual intensidade; e c) se existe ou não assimetria na transmissão de preços (AGUIAR, 1993).

O processo de transmissão de preços fundamenta-se nas hipóteses de que o movimento de preços e o ajustamento integral resultam em transmissão completa em algum ponto de tempo; na dinâmica e velocidade de ajustamento; e na assimetria de respostas, isto é, se as variações de preços são transmitidas assimetricamente entre os produtos. 


\section{METODOLOGIA}

\subsection{ENQUADRAMENTO METODOLÓGICO}

Para a realização do presente estudo, foi feita uma pesquisa de caráter quantitativo descritivo por meio de dados secundários coletados nas bases de dados do Cepea (Centro de Estudos Avançados em Economia) e IEA (Instituto de Economia Agrícola do Estado de São Paulo). Da pesquisa, resultaram as séries de preço mensal da cana-de-açúcar, do etanol hidratado e do açúcar no período de fevereiro de 1999 até setembro de 2010, sendo que todos os dados foram coletados no Estado de São Paulo.

A base de preço para o etanol hidratado é apresentada em $\mathrm{R} \$ /$ litro,

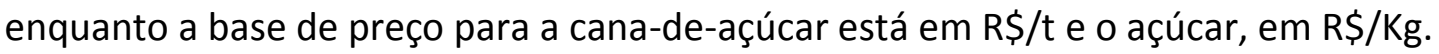

\subsection{MÉTODO DE INTERVENÇÃO}

Em análise de regressão múltipla, as inter-relações entre variáveis econômicas geralmente exigem que os modelos sejam tratados pela técnica de equações simultâneas. Nesses modelos, as variáveis são referidas como endógenas, exógenas ou predeterminadas, e a estimação delas exige alguns cuidados relacionados aos pressupostos, ou seja, existe restrições para estimação de modelos com equações simultâneas.

O primeiro passo foi verificar a existência de cointegração entre as séries por meio do procedimento proposto por Engle e Granger (1987), visando a auferir se existe a presença de um equilíbrio de longo prazo, ou seja, uma relação estável entre o conjunto de variáveis. Para a verificação da cointegração, é elaborada uma equação de regressão para o conjunto de variáveis da seguinte forma:

$y_{t}=\alpha+\alpha_{1} x_{1}+\alpha_{2} x_{2}+u_{t}$

Em que $x_{t}$ e $y_{t}$ são ditos cointegrados se existe parâmetro $\alpha$ tal que 
$u_{t}=y_{t}-\alpha x_{t}$

é um processo estacionário.

Onde $y_{t}$ e $x_{n}$ é o log-retorno do preço de uma das variáveis, $\alpha_{n}$ é o parâmetro calculado e ut é o resíduo da regressão.

Para tanto, foi efetuado o teste Dickey-Fuller Aumentado (ADF). Caso seja possível rejeitar a hipótese nula de não estacionariedade dos resíduos da relação de longo prazo entre cada par de variáveis em nível, é possível afirmar que as séries são cointegradas.

De acordo com Hill (2012), ao se trabalhar com séries temporais, evidenciase sempre a necessidade de estacionariedade da série. Sendo assim, o teste DickeyFuller Aumentado foi novamente utilizado para verificar a estacionariedade das séries. O teste ADF é repetido, quando necessário, fazendo-se diferenças sucessivas da série. O número de raízes unitárias (ordem de integração) é dado pelo número de vezes que a série deve ser diferenciada para se tornar estacionária.

Um processo estocástico é estacionário quando a sua média e a sua variância são constantes ao longo do tempo e quando o valor da covariância entre dois períodos de tempo depende apenas da distância, do intervalo ou da defasagem entre os períodos de tempo, e não do próprio tempo em que a covariância é calculada. Em termos de notação matemática, as propriedades do processo estocástico estacionário podem ser representadas por: (Média) $E\left(Y_{t}\right)=\mu$, (Variância) $\operatorname{var}\left(Y_{t}\right)=E\left(Y_{t}-u\right)^{2}=\sigma^{2} \mathrm{e}$ (Covariância) $\gamma_{k}=E\left[\left(Y_{t}-\mu\right)\left(T_{t+k}-\mu\right)\right]$ (BUENO, 2011).

O teste ADF consiste na estimação da equação por mínimos quadrados ordinários e pode ser expresso, conforme Tsay (2010), da seguinte forma:

$$
x_{t}=c_{t}+\beta x_{t-1}+\sum_{i=1}^{p-1} \phi_{i} \Delta x_{t-i}+\varepsilon_{t}
$$

onde $c_{t}$ é uma função determinística do tempo(t) e $\Delta x_{j}=x_{j}-x_{j-1}$ são as séries diferenciadas de $x_{t}$. A razão $t$ de $\hat{\beta}-1$,

$$
\text { ADF-test }=\frac{\hat{\beta}-1}{s t d(\hat{\beta})},
$$


onde $\hat{\beta}$ denota os mínimos quadrados estimados de $\beta$, é o teste de raiz unitária "aumented Dickey-Fuller" (ADF).

O modelo de Autorregressão Vetorial (VAR) foi proposto como alternativa aos modelos estruturais multiequacionais. Ele tem uma abordagem bastante utilizada na análise de questões macroeconômicas e tem sido também usado em estudos relacionados à economia agrícola, podendo-se citar, nesse caso, Myers et al. (1990), Aguiar (1993), Barros (1997) e Bacchi e Burnquist (1999).

O modelo VAR, proposto por Sims (1980), é constituído de séries temporais em que as variáveis dependentes estão em função de suas próprias defasagens e de outras variáveis. Ele pode ser escrito, em sua forma reduzida e de primeira ordem, pela seguinte forma matricial:

$$
y_{t}=\left[\begin{array}{l}
y_{1 t} \\
y_{2 t} \\
y_{3 t}
\end{array}\right]=\left[\begin{array}{l}
m_{1} \\
m_{2} \\
m_{3}
\end{array}\right]+\left[\begin{array}{l}
a_{11} a_{12} a_{13} \\
a_{21} a_{22} a_{23} \\
a_{31} a_{32} a_{33}
\end{array}\right]\left[\begin{array}{l}
y_{1, t-1} \\
y_{2, t-1} \\
y_{3, t-1}
\end{array}\right]+\left[\begin{array}{l}
\varepsilon_{1 t} \\
\varepsilon_{2 t} \\
\varepsilon_{3 t}
\end{array}\right]=m+A y_{t-1}+\varepsilon_{t}
$$

ou, de modo equivalente, como o seguinte sistema de equações:

$$
\begin{aligned}
& y_{1 t}=m_{1}+a_{11} y_{1, t-1}+a_{12} y_{2, t-1}+a_{13} y_{3, t-1}+\varepsilon_{t} \\
& y_{2 t}=m_{2}+a_{21} y_{1, t-1}+a_{22} y_{2, t-1}+a_{23} y_{3, t-1}+\varepsilon_{t} \\
& y_{3 t}=m_{1}+a_{31} y_{1, t-1}+a_{32} y_{2, t-1}+a_{33} y_{3, t-1}+\varepsilon_{t}
\end{aligned}
$$

A utilização da metodologia VAR permite a obtenção de elasticidades de impulso para $\mathrm{k}$ períodos à frente. Essas elasticidades de impulso possibilitam a avaliação do comportamento das variáveis em resposta a choques (inovações) individuais em quaisquer dos componentes do sistema, podendo-se, assim, analisar, por meio de simulação, efeitos de eventos que tenham alguma probabilidade de ocorrer.

A principal vantagem no uso do VAR é que se trata de um modelo multivariado, dinâmico, que permite analisar a relação entre as variáveis ao longo do tempo, levando-se em consideração a interdependência delas. 


\subsection{PROCEDIMENTOS DE PESQUISA}

Conforme descrito, utilizaram-se séries históricas de preços da cana-deaçúcar, etanol e açúcar, negociadas no Estado de São Paulo por ser o maior produtor nacional. As séries referem-se ao período de fevereiro de 1999 até setembro de 2010, totalizando 170 observações.

Com o objetivo de suavizar as séries de dados, a fim de dar maior clareza à análise do comportamento apresentado por elas, será utilizada, neste trabalho, a série em logaritmo natural, expresso nas variáveis "cane", "sugar", "etanolhidr.", representando os logaritmos dos preços da cana-de-açúcar, do açúcar e do etanol hidratado, respectivamente. As séries também foram diferenciadas, pois se trabalhou com os retornos dos preços a fim de verificar se as variações nos preços de uma ou mais variáveis produziriam alterações nos preços das demais variáveis. Este procedimento também visa a respeitar o pressuposto de estacionariedade conforme já disposto.

\section{ANÁLISE E DISCUSSÃO DOS RESULTADOS}

Seguindo o modelo analítico proposto, as séries de preço foram analisadas a fim de verificar a presença de raiz unitária e cointegração nas variáveis para que se pudesse dar início às estimativas do modelo VAR.

O teste de cointegração Engle-Granger foi utilizado para verificar se haveria uma relação de logo prazo entre as variáveis. O teste verifica a presença de raiz unitária nas variáveis em nível e realiza uma regressão entre elas para analisar se os resíduos da regressão possuem raiz unitária. 
Tabela 1 - Teste de cointegração de Engle-Granger

\begin{tabular}{lccc}
\hline Variável & Estatística do Teste & Valor crítico $5 \%$ & $\mathrm{p}$-valor Z(t) \\
\hline Cane & -1.438 & $-2,887$ & 0.5639 \\
Sugar & -1.603 & $-2,887$ & 0.4821 \\
Etanolhidr & -1.679 & $-2,887$ & 0.4420 \\
$u_{1}$ & -2.541 & -2.887 & 0.1059 \\
\hline
\end{tabular}

Fonte: Elaborado pelos autores, 2012.

Conforme se pode constatar pela análise da Tabela 1 , os resíduos $\left(u_{1}\right)$ da regressão possuem raiz unitária, assim como as variáveis em nível. Sendo assim, confirma-se a inexistência de cointegração entre os dados.

Para a realização da análise pelo modelo VAR, é fundamental que as séries utilizadas não possuam raiz unitária, ou seja, sejam estacionárias. Para tanto, as séries foram diferenciadas, gerando as variáveis "dcane", "dsugar" "detanolhidr", e foi realizado o teste Dick-Fuller Aumentado (ADF), conforme a Tabela 2.

Tabela 2 - Teste ADF para raiz unitária

\begin{tabular}{lccc}
\hline Variável & Estatística do Teste & Valor crítico 5\% & p-valor Z(t) \\
\hline Dcane & $-8,219$ & $-2,887$ & 0,0000 \\
Dsugar & $-8,346$ & $-2,887$ & 0,0000 \\
Detanolhidr & $-8,343$ & $-2,887$ & 0,0000 \\
\hline
\end{tabular}

Fonte: Elaborado pelos autores, 2012.

Confirmada a estacionariedade da série, partiu-se para a definição do número de defasagens a serem utilizadas no modelo.

Utilizou-se um sistema VAR com duas defasagens baseadas no critério Akaike de seleção. Os asteriscos na tabela indicam os melhores (isto é, minimizados) valores dos respectivos critérios de informação $\mathrm{AIC}=$ critério de Akaike, $\mathrm{BIC}=$ critério Bayesiano de Schwarz, e HQC = critério de Hannan-Quinn.

Tabela 3 - Critério de escolha do número de defasagens

\begin{tabular}{llllllll}
\hline Lag & $\mathrm{LL}$ & $\mathrm{LR}$ & $\mathrm{p}$ & $\mathrm{FPE}$ & $\mathrm{AIC}$ & $\mathrm{HQIC}$ & $\mathrm{SBIC}$ \\
\hline 0 & 536.445 & & & $7.4 \mathrm{e}-08$ & $-7,90289$ & $-7,87666$ & $-7,83833$ \\
1 & 565.674 & 58.457 & 0.000 & $5.5 \mathrm{e}-08$ & $-8,20257$ & $-8.09763^{*}$ & $-7.94433^{*}$ \\
2 & 578.556 & $25.765^{*}$ & 0.002 & $5.2 \mathrm{e}-08^{*}$ & $-8.26009^{*}$ & $-8,07643$ & $-7,80816$ \\
3 & 584.539 & 11.966 & 0.215 & $5.4 \mathrm{e}-08$ & $-8,21539$ & $-7,95303$ & $-7,56978$ \\
4 & 591.127 & 13.176 & 0.155 & $5.6 \mathrm{e}-08$ & $-8,17966$ & $-7,83859$ & $-7,34035$ \\
\hline
\end{tabular}

Fonte: Elaborado pelos autores, 2012. 
A análise da Tabela 3, pelo critério AIC, permite concluir que há influência do preço de um produto sobre o outro com até dois períodos anteriores (duas defasagens). A primeira variável analisada, "dcane", demonstrou sofrer influência de si própria em duas defasagens bem como da variável "detanolhidr" em uma defasagem, conforme tabela a seguir.

Tabela 4 - Modelo VAR para variável "dcane"

\begin{tabular}{lcccc}
\hline Termos & Coeficiente & Erro padrão & $\mathrm{z}$ & $\mathrm{p}$-valor \\
\hline dcane_1 & 0,160622 & 0,0848221 & 1,894 & 0,0605 \\
dcane_2 & 0,230326 & 0,0821562 & 2,804 & $0,0058^{* * *}$ \\
dsugar_1 & 0,0783095 & 0,0522727 & 1,498 & 0,1365 \\
dsugar_2 & 0,0562602 & 0,0510602 & 1,102 & 0,2726 \\
detanolhidr_1 & 0,0909898 & 0,0270254 & 3,367 & $0,0010^{* * *}$ \\
detanolhidr_2 & $-0,0205257$ & 0,0280330 & $-0,7322$ & 0,4654 \\
Const & 0,00298436 & 0,00306435 & 0,9739 & 0,3319 \\
\hline
\end{tabular}

Fonte: Elaborado pelos autores, 2012.

Observa-se que a variável "dsugar", por não ser significativa, não foi capaz de influenciar a "dcane". Desta forma, constata-se que o retorno do preço da cana-deaçúcar sofre influência positiva de si própria com dois períodos de defasagem e também é positivamente influenciado pelo retorno do preço do etanol hidratado. Porém, as variações no preço não são integralmente repassadas entre os produtos.

A Tabela 5 relaciona a equação proposta para a variável "dsugar".

Tabela 5 - Modelo VAR para variável "dsugar"

\begin{tabular}{lrrrr}
\hline Termos & Coeficiente & Erro padrão & \multicolumn{1}{c}{ Z } & \multicolumn{1}{c}{-valor } \\
\hline dcane_1 & 0,200564 & 0,140642 & 1,426 & 0,1562 \\
dcane_2 & 0,027982 & 0,136221 & 0,2054 & 0,8376 \\
dsugar_1 & 0,215902 & 0,086672 & 2,491 & $0,014^{* *}$ \\
dsugar_2 & $-0,08862$ & 0,084662 & $-1,047$ & 0,2972 \\
detanolhidr_1 & 0,089575 & 0,04481 & 1,999 & $0,0477^{* *}$ \\
detanolhidr_2 & 0,116303 & 0,046481 & 2,502 & $0,0136^{* *}$ \\
Const & 0,005138 & 0,005081 & 1,011 & 0,3138 \\
\hline
\end{tabular}

Fonte: Elaborado pelos autores, 2012.. 
Analisando a Tabela 5, pode-se concluir que o processo de transmissão de preços ocorre nas variáveis estudadas, no sentido de que o preço do açúcar é positivamente influenciado pelo retorno do próprio preço com uma defasagem, mas também é influenciado pelo retorno do preço do etanol hidratado com uma e duas defasagens. Ou seja, o preço do açúcar responde positivamente a elevações no preço do etanol, corroborando com os estudos de Silva e Almeida (2006), demonstrando haver uma ligação entre o preço do açúcar e dos combustíveis passando pelo etanol.

Cabe salientar que a variável "dsugar" não sofreu influência significativa da variável "dcane", ou seja, não se verificou o processo de transmissão de preços entre estes produtos. A última variável analisada, "detanolhidr", não demonstrou sofrer influência das demais variáveis, apenas de si própria.

Tabela 6 - Modelo VAR para variável "detanolhidr"

\begin{tabular}{lrrrr}
\hline Termos & Coeficiente & Erro padrão & Z & \multicolumn{1}{c}{-valor } \\
\hline dcane_1 & $-0,32049$ & 0,270646 & $-1,184$ & 0,2385 \\
dcane_2 & $-0,19848$ & 0,26214 & $-0,7572$ & 0,4503 \\
dsugar_1 & 0,070715 & 0,166789 & 0,424 & 0,6723 \\
dsugar_2 & 0,281177 & 0,16292 & 1,726 & 0,0867 \\
detanolhidr_1 & 0,360513 & 0,086231 & 4,181 & $0,0000^{* * *}$ \\
detanolhidr_2 & $-0,18573$ & 0,089446 & $-2,076$ & $0,0398^{* *}$ \\
Const & 0,010116 & 0,009778 & 1,035 & 0,3028 \\
\hline
\end{tabular}

Fonte:Elaborado pelos autores, 2012.

Nesse sentido, a transmissão de preços não foi verificada entre o etanol hidratado e as demais variáveis. Porém, o retorno do preço do etanol hidratado demonstra sofrer forte influência apenas do seu próprio preço, com um e dois períodos de defasagem.

Merece grande destaque o fato de o etanol hidratado não sofrer influência dos demais produtos da cadeia, mas ser capaz de influenciar todos os demais. Isso demonstra que o etanol hidratado tem comportamento de líder na cadeia, e as variações nos seus preços são transmitidas aos demais. 
A transmissão de preço se dá, portanto, do etanol para a cana-de-açúcar e para o açúcar, com um período de defasagem para a cana-de-açúcar, e com um e dois períodos para o açúcar.

O processo de transmissão de preços dentro do setor sucroalcooleiro ocorre de forma assimétrica entre os produtos estudados. Apesar de efetivo, o processo de transmissão de preço é insuficiente para validar a LPU dentro do setor, tendo em vista que as alterações e as variações de preços são transmitidas assimetricamente e não são repassadas de forma integral aos demais produtos. Conforme Fackler e Goodwin (2001), os pressupostos teóricos da LPU são bastante restritivos e não consideram outras variáveis importantes na interação.

\section{CONSIDERAÇÕES FINAIS}

O setor sucroalcooleiro mostra-se como um dos mais dinâmicos do agronegócio brasileiro. Merece destaque neste setor a cadeia do etanol, que se relaciona tanto com o mercado de combustíveis quanto com o de alimentos, por meio do açúcar.

O presente estudo evidencia a importância da pesquisa das relações de preço entre os biocombustíveis e os alimentos. Ambos, alimentos e combustíveis, são setores estratégicos da economia, e suas alterações de preços têm impacto importante em diversos setores, inclusive como componentes no cálculo da taxa de inflação.

O processo de transmissão dos preços demonstrou-se efetivo, havendo integração entre os preços dos produtos estudados. Com os resultados, foi possível verificar o sentido da transmissão dos preços e a intensidade com a qual ocorre.

A análise de transmissão de preços dentro do setor sucroalcooleiro demonstrou haver integração entre os preços das variáveis estudadas. Tanto o preço da cana-de-açúcar quanto o do açúcar sofreram influência significativa do preço do 
etanol hidratado, porém nenhum daqueles produtos foi capaz de influenciar os preços deste. Esse comportamento evidencia o poder que os biocombustíveis têm sobre outros elementos de sua cadeia produtiva e em destaque sobre os alimentos.

O preço do etanol demonstrou sofrer influência apenas de si próprio, com um e dois períodos de defasagem, evidenciando, assim, que o etanol é o líder da cadeia de produtos estudada, sendo capaz de determinar variações nos preços dos demais produtos. Dessa forma, as políticas para este combustível merecem atenção, pois determinarão reflexos em toda a cadeia e, por consequência, no setor sucroalcooleiro como um todo.

Apesar de efetivo o processo de transmissão de preço dentro do setor, não foi validada a Lei do Preço Único como mecanismo de ajuste de preços, pois as alterações e as variações de preços são transmitidas assimetricamente e não são repassadas de forma integral aos demais produtos, contrariando o pressupostos da teoria.

O estudo sofre algumas limitações, tendo em vista que há outros produtos e componentes importantes na formação de preços na cadeia estudada. Sugere-se, para estudos posteriores, que se incluam preços dos demais combustíveis, em especial o da gasolina, pois esta recebe a adição de $20 \%$ de etanol em sua mistura, podendo-se assim verificar a interação deste produto com a cadeia do etanol. Uma alternativa a ser explorada é a utilização de modelos multivariados que incluam tendências como o threshold e, assim, verificar o comportamento que estas variáveis assumem quando há variações nas tendências.

PRICE TRANSMISSION ANALYSIS IN BRAZILIAN SUGARCANE SECTOR

\section{ABSTRACT}

Aims of this paper is to analyze price transmission process in sugarcane sector and verify the applicability of Law of One Price. For this, it was used price series of 
sugarcane, hydrated ethanol and sugar in the state of São Paulo from February 1999 to September 2010. These data were analyzed according to the Vector Autoregressive Model. The results found show a strong link among these products, highlighting the case of hydrous ethanol, which strongly influences the prices of the other ones, but does not suffer any influence from fluctuations in the prices of sugarcane and sugar.

Key-words: Price transmission. Sugarcane. Ethanol. Sugar.

\section{REFERÊNCIAS}

AGUIAR, D. R. D. A questão da transmissão de preços agrícolas. Revista de Economia e Sociologia Rural, Brasília, v.31, n.4, p.291-308, out./dez., 1993.

AGÊNCIA NACIONAL DO PETRÓLEO, GÁS NATURAL E BIOCOMBUSTÍVEIS. Anuário ANP 2011. Disponível em: <http://www.anp.gov.br/?pg=57890>. Acesso em: 28 maio 2012.

ARDENI, P. G. Does the Law of One Price really hold for commodity prices? American Journal of Agricultural Economics, v. 71, n.3, p. 661-669, 1989.

BACCHI, M.R.P.; BURNQUIST, H.L. Transmissão de preços entre os segmentos produtivos da pecuária de corte brasileira. In: CONGRESSO BRASILEIRO DE ECONOMIA E SOCIOLOGIA RURAL, Foz de Iguaçu, PR, 1999. Anais... Brasília: SOBER, 1999.

BALCOMBE, B. K.; MORRISON, J. Commodity price transmission: a critical of techniques and an application to select tropical export commodities. Roma: FAO, 2002.

BARROS, G.S. de C.; BITTENCOURT, M.V.L. Formação de preços sob oligopsônio: o mercado de frango em São Paulo. Revista Brasileira de Economia, Rio de Janeiro, v.51, n.2, p.181-199, 1997.

BUENO, R. D. L. D. Econometria de séries temporais. São Paulo: Cengage Learning, 2011.

CANÊDO-PINHEIRO, M. (2011) Assimetrias na Transmissão dos Preços dos Combustíveis: O Caso do Óleo Diesel no Brasil. FGV-IBRE, Textos para discussão. Disponível em:

<http://portalibre.fgv.br/main.jsp?lumPageld=402880811D8E34B9011D9CCBFDD1784 C\& contentld=8A7C82C53186E3FA0131AE7CD590679D>. Acesso em 30 maio 2012. 
COSTA, C.C. da; BURNQUIST, H.L.; GUILHOTO, J.J.M. Impacto de alterações nas exportações de açúcar e álcool nas regiões Centro-Sul e Norte-Nordeste sobre a economia do Brasil. Revista de Economia e Sociologia Rural, v.44, n.4, p. 609-627, 2006.

DICKEY, D.A.; FULLER, W.A. Likelihood ratio statistics for auto-regressive time series with a unit root. Econometrica, v.49, n.4, p.1057-72.1981.

ENGLE, R.;GRANGER, C. Cointegration and error correction: representation, estimation and testing. Econometrica, v.55, n. 2, p. 251-276, 1987

ELOBEID, A.; TOKGOZ, S.; HAYEZ, D. J.; BADCOCK, B. A.; HART, C. E.; The Long-Run Impactof Corn-Based Ethanol on the Grain, Oilseed, and Livestock Sectors: A Preliminary Assessment. CARD Briefing Paper 06-BP 49. November 2006.

FACKLER, P. L; GOODWIN, B. K. Spatial price transmission. In: GARDNER, B.; RAUSSER, G. (Eds.). Handbook of agricultural economics. Amsterdam: Elsevier, 2001.

FULLER, W.A. Introduction to statistical time series. New York: John Wiler. 1976.

GAMARRA, J. Transmissão de preços entre os mercados de etanol e da gasolina desde o lançamento dos carros Flex-Fuel no mercado brasileiro. 2009. 143 f. Dissertação (mestrado em Agronegócio) Universidade Federal do Rio Grande do Sul, 2009.

HILL, R.C.; GRIFFITHS, W.E.; LIM, G.C. Principles of Econometrics. 4. ed., New York: John Wiley \& Sons. 2012.

JORNALCANA. Conheça o setor. Disponível em: <http://www.jornalcana.com.br/Conteudo/Conheca\%20o\%20Setor. asp>. Acesso em: 18 abril 2011.

KRUGMAN, P. R.; OBSTFELD, M. International economics: theory and policy. 5. ed. Massachusetts: Addson Welsley, 2005.

MYERS, R.J.; PIGGOTT, R.R.; TOMEK, W.G. Esthimating sources of fluctuations in the australian wool market: an application of VAR methods. Australian Journal of Agricultural Economics, v.34, p.242-262, 1990.

SILVA, C. M. S; ALMEIDA, E. L. F.Formação de um mercado internacional de etanol e suas inter-relações com os mercados de petróleo e açúcar. In: CBE, 16 a 18 de agosto de 2006, Rio de Janeiro.

SIMS, C.A. Macroeconomics and reality. Econometrica, v. 48, n.1, p. 1-48. 1980. 
TSAY, RUEY S. Analysis of Financial Time Series, 3 ed. New York: John Wiley \& Sons, Inc., 2010.

TOKGOZ, S., ELOBEID, A.; J.FABIOSA, D.J.HAYES, B.A. BADCOCK, C.E. HART, T. YU, F. DONG, J.C. BEGHIN. 2007. Emerging biofuels: outlook of effects on U.S. grain, oilseed, andlivestock markets. CARD Staff Report 07-SR 101, May, Center for Agricultural and Rural Development, Ames, IA, 2007.

VIDOSH, M. PRAKSH V. ALOK, C. Impacts of Bio-fuel use: A Review, International journal of engineering science and technology. v. 3 n. 5, 2011. 